\title{
General Spectral Camera Lens Simulation
}

\author{
B. Steinert and H. Dammertz and J. Hanika and H. P. A. Lensch
}

Ulm University, Germany

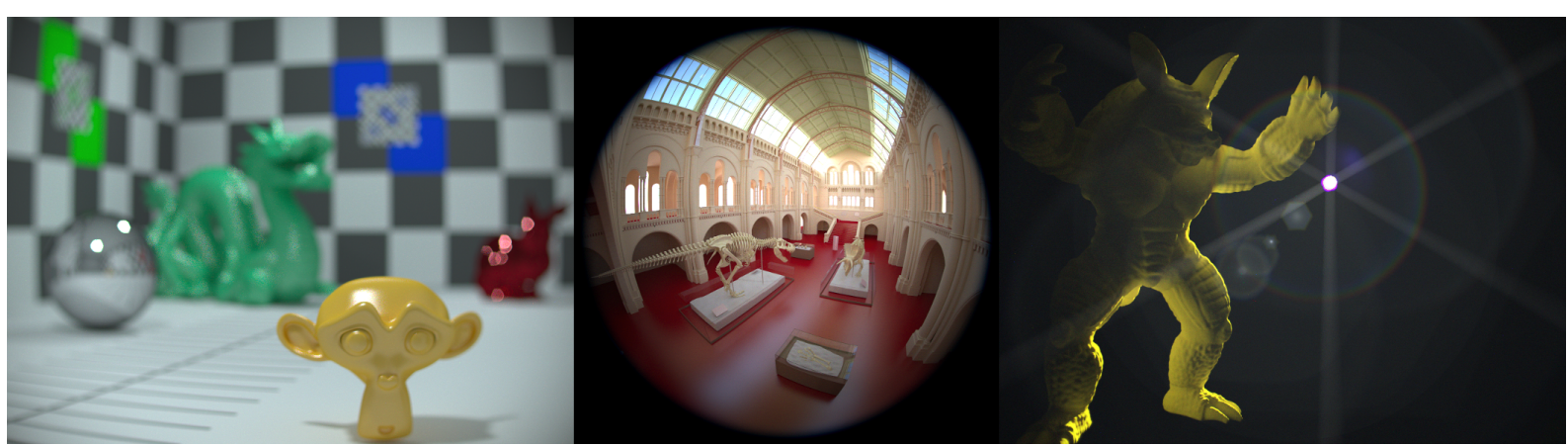

Figure 1: Three images created with our lens simulation model. From left to right: realistic depth of field and bokeh, fisheye distortion, lens flare artifacts and glare streaks.

\begin{abstract}
We present a camera lens simulation model capable of producing advanced photographic phenomena in a general spectral Monte Carlo image rendering system. Our approach incorporates insights from geometrical diffraction theory, from optical engineering, and from glass science. We show how to efficiently simulate all five monochromatic aberrations, spherical and coma aberration, astigmatism, field curvature, and distortion. We also consider chromatic aberration, lateral color and aperture diffraction. The inclusion of Fresnel reflection and lens coatings generates correct lens flares and we present an optimized sampling method for path generation.
\end{abstract}

Categories and Subject Descriptors (according to ACM CCS): I.3.7 [Computer Graphics]: Three-Dimensional Graphics and Realism-Color, shading, shadowing, and texture

\section{Introduction}

The synthesis of photo-realistic images with today's techniques delivers high quality pictures with an outstanding degree of scene realism. However, some real-world photographic effects such as realistic depth of field, vignetting or distortion are often neglected. To include these effects realistically in computer graphics, light transport has to be simulated as if there was a real camera lens forming the image. In order to search for new realistic approximations or to validate existing ones, a reference simulation is needed which behaves like a real optical device. Our approach is able to reproduce all relevant optical monochromatic aberrations, and, additionally, chromatic aberrations due to full continuous spectral simulation. Accounting for the Fresnel equations at every single lens surface, lens flares can be formed. The inclusion of the geometrical theory of diffraction [Kel62] al- lows for the extension to simulate glare streaks caused by diffraction at the aperture blades. Additionally, a highly efficient sampling method for path generation through a lens is proposed.

\section{Related Work}

\subsection{Camera Models}

One of the primary goals of computer graphics is to produce realistic images by modelling reality. The camera model plays an important role in how the final image is produced. This becomes especially important in movie production where synthetic images are combined with real images in which for example the depth of field is used as element in the story. Thus depth of field simulation [CPC84] and fast approximations [MK06] have been studied in great de- 
tail. The pinhole camera model commonly used in computer graphics for its simplicity and ease of evaluation does not even support depth of field. It renders sharp, distortion free images with an infinite depth of field [Shi00]. The thick lens model [KMH95] provides a linear approximation with regard to the lens thickness, causing significant visual influence by optical path length manipulation [PP06]. It originates from optical design analysis [Smi07]. The linear transformation based on paraxial optics allows to express the imaging process of a whole system of lenses in a single transformation matrix. The thin lens model can be directly derived from the thick lens approximation by reducing lens thickness to zero. In contrast, we directly simulate the whole lens system and thus include more optical effects.

\subsection{Physically Based Light Transport Simulation}

In photo-realistic image synthesis one generally solves the rendering equation [Kaj86] in order to produce an image. Algorithms to solve this equation have been discussed in detail [PH04] and one common solution is to use Monte Carlo light transport simulation as described by Veach [Vea97]. As our goal is to capture as many optical effects as possible we need to account for the wavelength of light as another dimension in Monte Carlo integration. The actual image is formed by sampling a sensor plane and starting paths traversing an optical device.

\subsection{Full Lens Systems}

Established models like the thin and thick lens model are not able to simulate sophisticated optical effects like lens aberrations of higher order, or flare effects inside the system. In [KMH95], a complete realistic simulation of the imaging process was suggested first in a distribution ray tracing setting. Our approach based on Monte Carlo path generation methods extends the proposals by certain important methods: wavelength dependent events, the use of real glass data and consideration of dielectric Fresnel interaction at every glass surface.

Moreover, we propose a sampling method to overcome the problem of nonlinear distortion of the exit pupil to efficiently sample the cone of illumination formed by the lens and the aperture gate. For the latter element we extend the model by simulating diffraction events by the geometrical theory of diffraction.

\subsection{Diffraction}

The complex wave property of light causes diffraction effects, not explainable with classic geometrical optics. Approaches by Joseph B. Keller [Kel62] adopted the wave behavior of light to a modified Fermat setting, enabling us to make statements on diffraction in a ray-based simulation setting. Aveneau and Mériaux proposed using this theory in an image rendering setting [AM99]. As we only want to simulate diffraction at the aperture gate we can significantly reduce the complexity of this procedure. Glare effects have been modelled by image-based approaches [SSZG95], but mainly considered the human visual system so far.

\section{Optical Fundamentals}

\subsection{Geometrical Theory of Diffraction}

The geometrical theory of diffraction [Kel62, MPM90] is a simulation approach based on Fermat's law with the following modification: "An edge-diffracted ray from a point $P$ to a point $R$ is a curve which has stationary optical length among all curves from $P$ to $R$ with one point $Q$ on the edge." This allows for a direct usage in a ray-based image generation setting. Avenau and Meriaux [AM99] already introduced the model by Keller to Computer Graphics for scenebased diffraction account of direct light. Light is diffracted near edges of obstacles. The diffraction event is determined by the geometry of the occluder. For our purposes, it is sufficient to look at diffraction on straight edges lying on a wedge with opening angle $\alpha$.

The explicit direction for one ray in a simulation step is determined by the Keller cone (shown in Figure 2) with opening angle $\beta^{\prime}$ equal to the angle of incidence $\beta$. It describes the set of all valid diffraction directions for a diffraction location $Q$. For perpendicular incidence on the edge with $\beta=90^{\circ}$ the cone degenerates to a disk around $Q$.

Diffracted radiance at a location $R$ can be evaluated by a diffraction coefficient for a known diffraction point and geometry. With given projected angles in Figure 2, the diffracted radiance from $P$ to $R$ over $Q$ on the wedge with $\alpha=\pi(2-n)$ is [AM99]:

$$
L_{d}=L_{i} \lambda\left(\frac{\cos \frac{\pi}{4} \sin \frac{\pi}{n}}{n \pi \sin \beta\left(\cos \frac{\pi}{n}-\cos \frac{\delta-\gamma}{n}\right)}\right)^{2}
$$

This formula is valid for unpolarized spherical wavefronts hitting a straight edge, neglecting interference. It is important to note that the contribution of diffracted rays and the rest of the image, created with standard geometrical optics, can simply be summed up to obtain the final image.

\subsection{Glass Interaction}

The correct simulation of light interacting with glass surfaces is of paramount importance for the following suggestions. [DCWP02] provides an overview over all necessary formulas. Based on this, we use Sellmaier definitions of typical optical glass types that can be found in the Schott glass catalog [Sch09]. 


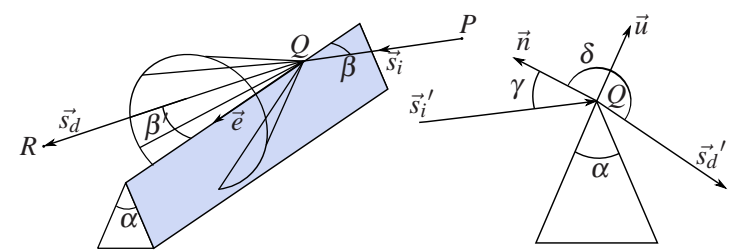

Figure 2: The cone of diffracted rays with the diffraction edge as axis and opening angle $\beta=\beta^{\prime}$ stated by Keller. The edge is settled on a wedge with inner angle $\alpha$. By projecting into the plane perpendicular to the diffraction edge (right) $\gamma$ and $\delta$ are introduced, which lie between the normal of the hit wedge plane and the projected vectors ${\overrightarrow{s_{i}}}^{\prime}$ and ${\overrightarrow{s_{d}}}^{\prime}$. Direction $\vec{u}$ completes the edge coordinate system with $\vec{e}$ and $\vec{n}$.

\subsection{Aberrations}

In the lens the varying thickness and incident angle due to the spherical surface causes a changing optical path length with varying ray height $h$ (see Figure 3), i.e. distance of the ray to the optical axis. Consequently, the image suffers from so-called Seidel aberrations [Ray02, Smi07]. Tracing rays through an optical system means applying Snell's law [DCWP02] repeatedly:

$$
\eta(\lambda) \sin \theta=\eta^{\prime}(\lambda) \sin \theta^{\prime}
$$

The Taylor expansion of $\sin \theta$ reveals that the incident angle appears in different orders.

$$
\sin \theta=\sum_{n=0}^{\infty}(-1)^{n} \frac{\theta^{2 n+1}}{(2 n+1) !}=\theta-\frac{\theta^{3}}{3 !}+\frac{\theta^{5}}{5 !} \pm \cdots
$$

Discarding all terms of higher order than the first yields the paraxial approximation

$$
\sin \theta \approx \theta
$$

which is valid for small angles $\theta$. This linear approximation of Equation 3 is the central basis of all linear lens models like the thin or thick lens description [PP06, Smi07]. By truncating the higher order terms, aberrations for a larger ray height with increasing $\theta$ can not be simulated anymore. In contrast, we use Snell's law (Equation 2) for our computations and therefore the ray height is not limited. Figure 3 depicts the difference between linear Gaussian optics and reality for marginal rays with large height $h$. Seidel [vS57] classi-

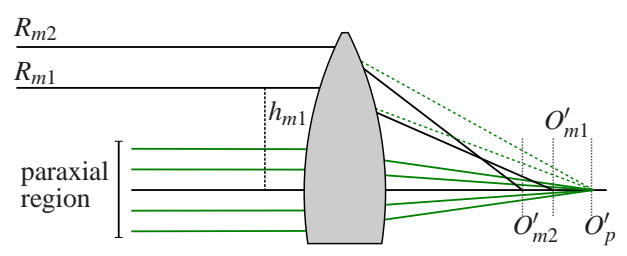

Figure 3: Comparison of Gaussian refraction (dashed) and Snell's refraction (solid) of marginal region rays.

fied five different forms of monochromatic aberrations introduced with the 3 rd order. The effect that paraxial rays with large height are focused closer to the lens is called spherical aberration. For oblique rays, coma aberration causes comet-like tails to points. Astigmatism is caused by the fact that the ray height can vary in two directions, resulting in different image planes. Field curvature is the obvious consequence of using spherical surfaces. The image is not formed on a real plane, but on a curved surface. Distortion arises with marginal rays, becoming more or less magnified, and thus introducing either inward pincushion distortion or outward barrel distortion. Chromatic aberrations are caused by dispersion during transmission. Shorter wavelengths are focused closer to the lens because of stronger refraction compared to longer wavelengths.

\subsection{Lens Design}

Optical instruments usually consist of one or more spherically curved, axially symmetric glass surfaces [Ray02]. Using lenses in tandem, either cemented or with a gap in between, offers possibilities for aberration correction, imaging distance or effective focal length manipulation. A typical description of a lens design is outlined in Figure 4.

\begin{tabular}{llll} 
radius & thickness & material & radius \\
\hline 42.970 & 9.8 & LAK9 & 19.2 \\
-115.33 & 2.1 & LLF7 & 19.2 \\
306.840 & 4.16 & air & 19.2 \\
aperture & 4.0 & air & 15.0 \\
-59.060 & 1.870 & SF7 & 17.3 \\
40.930 & 10.640 & air & 17.3 \\
183.920 & 7.050 & LAK9 & 16.5 \\
-48.910 & 79.831 & air & 16.5 \\
\hline
\end{tabular}

Figure 4: Tessar Design by Brendel (USP 2854889) [Smi05], $f / 2.8,100 \mathrm{~mm}$ effective focal length (EFL).

Each row describes one spherical surface inside the device. The curvature follows a fundamental rule: A positive radius stands for a surface with center of curvature on the right side, and negative sign means that the center is on the left side [Smi07]. The thickness applies to the transmission distance along the optical axis. The material name is a code referencing an entry in the Schott glass catalog [Sch09]. The last column represents the semi-diameter of the element. The aperture stop position and radius are defined in row 4. It is important to note that all values are scaled with the same factor when using the same lens design for a different focal length [Smi05].

Aperture Stop Irradiance transport in an optical system is controlled by a resizable diaphragm called aperture stop [PP06, Smi07, Ray02]. As outlined before, aberration critical rays near the margin can be blocked here. The entrance and exit pupils depend on aperture size and position. They can be regarded as images of the aperture on both sides of the lens system. The light forming character of a lens system can therefore be reduced to the two pupil disks, which allows concatenation of lens systems. 


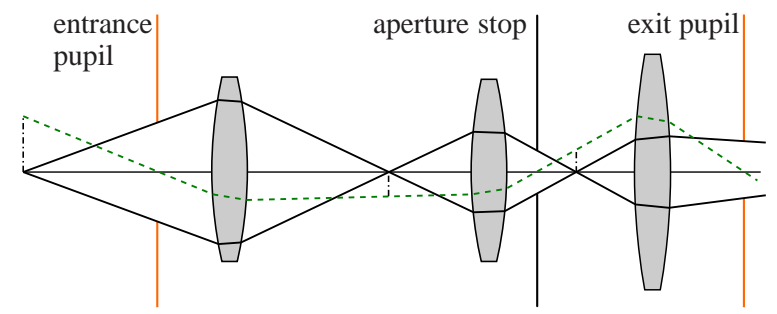

Figure 5: Adopted from [Smi07]. The green, dashed principal ray defines pupil locations, the axial ray fan determines the radii. The aperture stop is also treated as a pupil.

The size of the aperture is expressed by the photographic $f$-number (normally written as $f / N$ )

$$
N=\frac{f}{d_{\text {entr }}}
$$

with entrance pupil diameter $d_{\text {entr }}$ and effective focal length of the lens system $f$. The latter corresponds to the focal length of one single lens approximating the whole system.

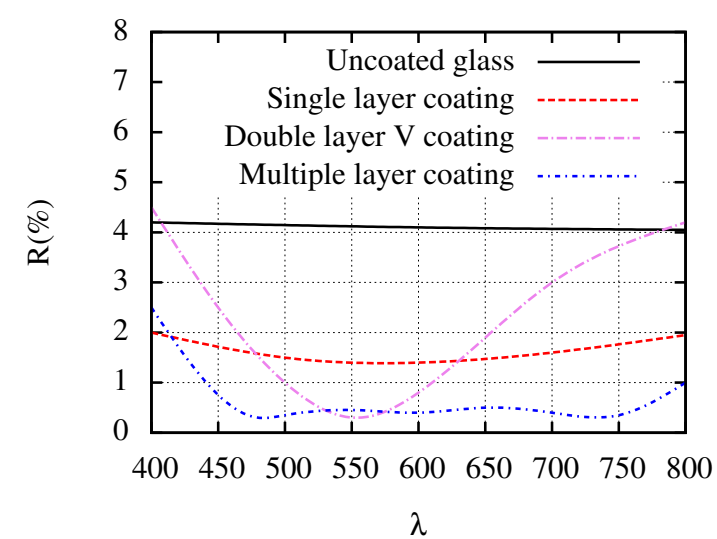

Figure 6: Comparison of reflectivity under normal incidence for different coatings [Ray02]. Coating stops a considerable amount of light from being reflected, allowing it to contribute to the image.

Material Coatings Ideally, an optical system delivers all incident light to the image plane. Light travelling from one medium to another will, however, be partially reflected. The amount of reflected light depends on the index difference. Thin film lens coatings are used to reduce reflection by intermediate layers. Example relations between different coatings and wavelengths are given in Figure 6.

\subsection{Secondary Effects}

In the following section, we will look at secondary effects that arise as soon as a lens design is used beyond its limits or is not sufficiently refined with coatings.
Flare Effects Light in a lens housing is not only refracted towards the sensor. Internal reflection cannot be completely eliminated and becomes clearly visible in certain situations. A typical flare effect is oblique incoming light, that gets reflected at a surface and then reaches the image plane [Smi07].
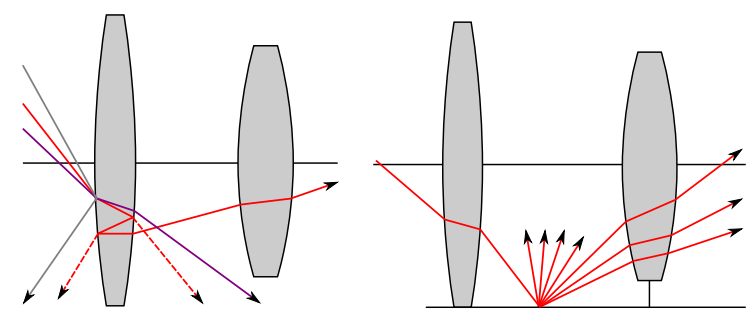

Figure 7: Secondary reflections inside the lens barrel can arise due to extreme angles of incidence, which cause internal reflections inside the elements, as seen on the left. The right schematic shows, that stray light occurs when the barrel has a certain reflectance.

Vignetting Inhomogeneous illumination of the imaging area is called vignetting and consists of three independent subtypes [Ray02]. Besides natural vignetting, caused by the varying angles of incidence and the projected pupil area for different sensor locations, mechanical vignetting is caused by obstacles like lens hoods. Optical vignetting is a con-

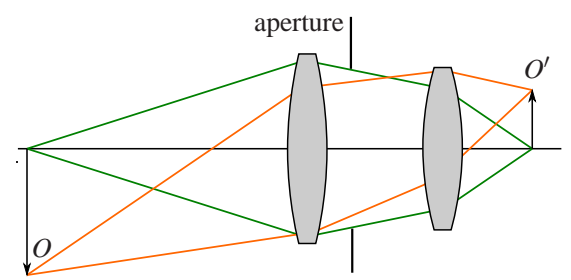

Figure 8: Optical vignetting is caused by the lens design. The aperture cannot be completely imaged because of the limited diameter of the lens in front and behind it.

sequence of off-axis rays having another effective aperture, because not all elements of the system can receive the full cone of illumination formed by the aperture [Smi07]. Figure 8 drafts two different cases. The reduced aperture area of oblique rays causes a respective decrease of irradiance at the image plane.

\section{Full Lens System for Realistic Image Synthesis}

\subsection{Implementation}

The new setting presented in this paper combines several well-understood concepts to yield a rendering system capable of accepting a real lens design. Basic implementation ideas are adopted from [KMH95]. Continuous spectral path evaluation was added to obtain chromatic artifacts. Spherical 

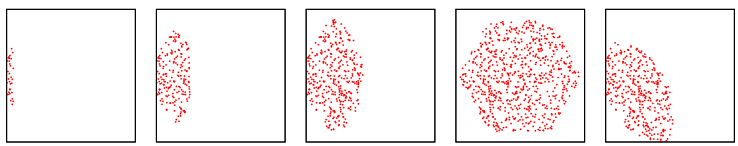

Figure 9: Sampling the image of the effective aperture for different pixel locations. The pixel pupils shows large distortion. The first four images show the positive sample locations in the pupil plane for pixel locations on the mid pixel row, starting at the fisheye characteristic illumination border ending at the center pixel of the sensor. The last image depicts the shape for a central pixel in the first quadrant of the sensor.

caps were chosen as analytic description for the lens design. All ray generation is isolated in a lens tracing kernel which is just used when trigger surfaces in front or in the end are hit. Direct light estimation [Shi00] is done by sampling these surfaces. Our system features flexible scales and precision to overcome numerical flaws. Scenes are represented in units of meters whereas millimeters are used for the lens tracing. This avoids the resolution problems of floating point arithmetic (to handle self-intersection, an offset of $0.001 \mathrm{f}$ is common). The aperture is described by the geometry of the opening. Scaling allows for setting any desired $f$-number.

\subsection{Pupil Sampling}

As can be seen in Figure 8, only paths passing through the effective aperture contribute to the image. Ideally, in a backward path tracing setting starting on the sensor, we only want to sample paths which pass through this aperture. This can be done by sampling the image of the effective aperture on a virtual plane between the image plane and the back lens, which we call pixel pupil. As the name indicates, this pupil depends on the pixel on the image plane.

The pixel pupil can be approximated by a circle, which is stored per pixel. The diameter and center are evaluated by a number of sample paths which are traced through the system. The mean of all intersections with the pupil plane yields the center, and the radius is chosen to include all points plus an optional, small offset. This approximation is used for all paths starting from somewhere inside the pixel. Figure 9 shows a few example pupils, Figure 10 illustrates the geometry of the pixel pupil, and Figure 11 visualizes the procedure. A nice side-effect can be observed for this fisheye lens: not all pixels on the image plane are illuminated. Using pixel pupil sampling, no paths are created for these pixels, as the aperture is completely occluded.

Further optimizations to the method can be easily added. For radially symmetric lenses, it is possible to exploit the symmetry and sample only one quadrant. Furthermore, as the number and order of spherical caps to intersect is given in advance, the pixel pupil data could be precomputed on the GPU for a high number of samples in very short time.

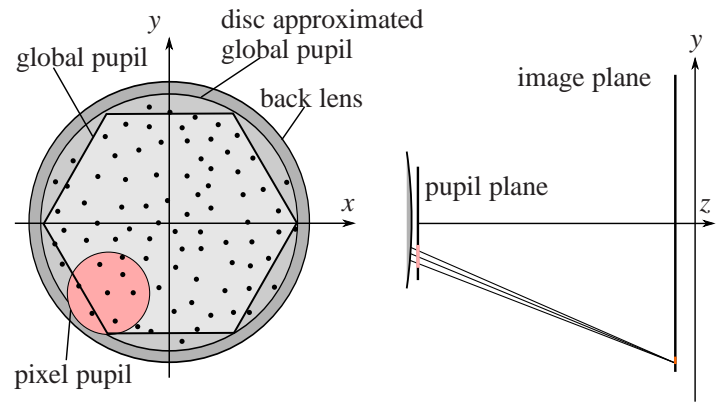

Figure 10: Samples of ray directions on the global pupil are stored if the constructed path reaches the other side. The average of the transmitted path samples yields the center for a pixel pupil. Because the circle is a rather coarse approximation, there is still a region off the pupil border which causes a small but acceptable amount of zero-contribution rays.
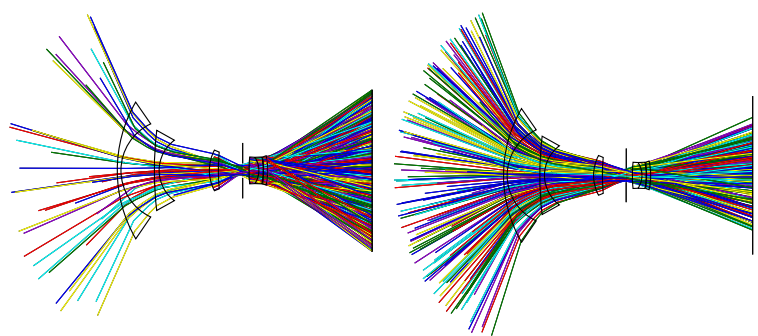

Figure 11: Lens tracing plot for comparison between global pupil sampling (left) and pixel pupil sampling (right) using the same number of initial rays, for a $10 \mathrm{~mm}$ Muller Fisheye lens at $f / 8$. In numbers, the ray passage rate is $10.2 \%$. By pixel pupil sampling, the number increases to averaged $79.7 \%$ per frame. By choosing an even more precise pupil representation this number could be optimized to nearly $100 \%$. An exactly computed pupil could indeed save the intersection test with the aperture stop.

To extend the proposed algorithm to a forward path tracing approach starting at light sources, a virtual image plane on the object side of the lens system is needed to discretize the field of view. In order to keep the area small it should be almost right in front of the lens. The computation of the pupils is now almost the same, except for one additional test if the sample reaches the image plane inside valid bounds.

The presented method does of course only apply to a setting where inner lens reflection is completely ignored. These effects are calculated by another method and added to the final result.

\subsection{Lens Flare Simulation}

Since a single ray-based rendering technique cannot sufficiently cover all possible effects, a progressive rendering method that combines several techniques is required. The most common is to use forward and backward ray tracing together in a bidirectional fashion using multiple impor- 

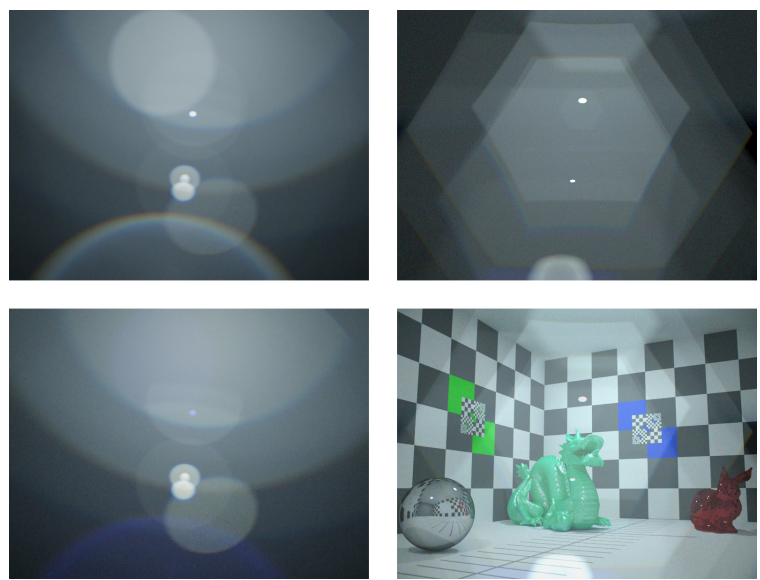

(a) Here, a $33 \mathrm{~mm}$ wide-angle lens by Mori [Smi05] at $f / 2.8$ was used for the left flare images. A lens surface was equipped with a modified reflection spectrum resembling a thin layer Vcoating [Smi07] (lower left). The right images show the lens flare result of of Kimura wide-angle with $f / 5.6$ clearly reproducing aperture ghosts. The combined image gives an impression of the resulting impact.
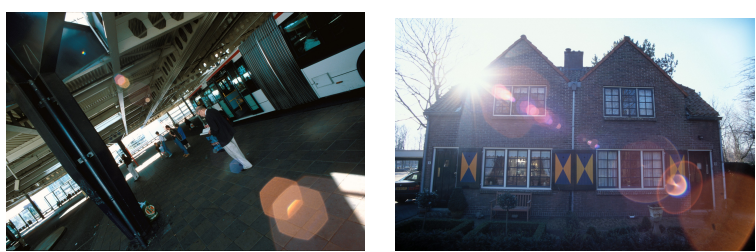

(b) Left: Reference photograph of a typical flare light situation with the source beeing outside the field of view. Right: Features different optical artifacts. First, noticeable distortion, glare streaks and halo around the sun, and outshining lens flares over the image. By courtesy of Paul van Walree [vW].

Figure 12: Inner reflections in lenses can be reduced by thin layer coating and thus introduce color artifacts by the absorption characteristic.

tance sampling [Vea97]. For the specific phenomenon of lens flares, we only need to consider direct light from the scene to obtain the light paths for reflection artifacts. Lens flares are best found by starting samples at light sources and deterministically connecting them to the lens. The resulting image delivers an independently viewable solution of the lens flare as shown in Figure 12a (left). The results are only correct for the predetermined setting of direct light. Mirror interactions are not accounted for in the light tracing pass but could be easily included by extending the light paths and filtering out appropriately in the backward stage. Neglecting direct light in the backwards path tracing stage allows both results to be summed up to the final image in Figure 12a (right). The lens tracing plot in Figure 13 clearly shows regions on the sensor, where reflected light arrives.

In order to model the typical color variations of lens flares, absorption characteristics of material coatings have to be

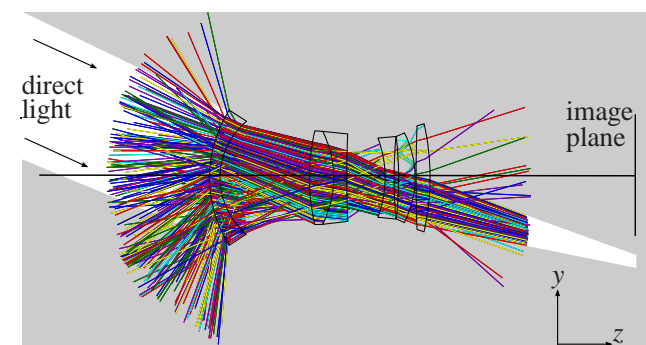

Figure 13: Lens tracing plot for an aperture-less Momiyama wide-angle [Smi05] at $36 \mathrm{~mm}$ with fix $f / 2.8$, simulating lens flare. either directly at the first surface or later. The image of the light source itself is not in the field of view. The reflected rays reaching the sensor create the flare effects.

simulated. We approximate thin-film interference by including a lookup step right after Fresnel computation. The degree of transmission is altered according to the desired coating with data from Figure 6 for specific surfaces.

\subsection{Aperture Diffraction}

Diffraction at the aperture causes significant glare streaks crossing the image when looking into bright lights. The geometrical theory of diffraction allows to introduce paths into the rendering setting which produce these effects. The diffraction direction must fulfill the Keller cone rules and has to start on the edge of the aperture, or at least very near to it, to be able to use the diffraction coefficient proposed in [AM99]. During lens tracing and aperture testing, we are able to check whether the ray almost hits the edge for example by testing the barycentric coordinates when using triangles. By choosing a certain interval around the edge, we can accept paths to be diffracted according to the rules stated above. The diffraction direction has to be sampled randomly on the Keller cone surface. By sampling a point on the base circle as direction from the edge hitpoint we receive a valid diffraction direction with probability $\frac{1}{\pi r^{2}}$. After that, the diffracted radiance can be computed using Equation 1 . For simplicity reasons we assume to have an infinitely thin aperture by applying $\alpha=0$. Diffraction in the aperture corners has not been accounted for in this work, but could be added by specific coefficient and direction calculations. Note that the diffraction process generally resembles a surface material interaction. Thus we implemented the process as diffraction BRDF.

\section{Results}

In the following, we present renderings of individual effects. Note that all effects could be present and correctly rendered in a single image with our system.

Aberration Simulation In order to simulate photorealistic aberration, our system applies Snell's law according to the continuous spectrum. Figure 14 outlines various aberrations 


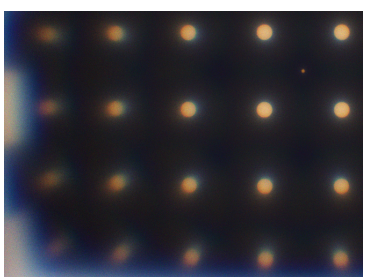

(a) Back wall in focus. Unequal focus near the border is caused by spherical aberration and field curvature. The comet-like tail at the marginal points is coma

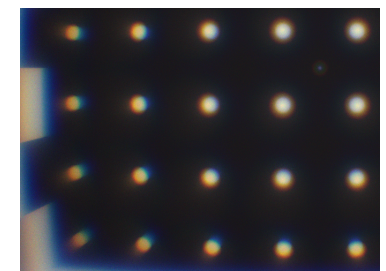

(b) Focus shifted a bit. The color shift of the marginal circles is called lateral color originating from varying magnification of all wavelengths. aberration.

Figure 14: These two images show the lower left of a shot through a single biconvex lens, which suffer all aberrations uncorrected. The small upper right point is the actual image center. Both images show significant distortion.

of a single spherical lens. The scene is moved in and out of focus. Chromatic artifacts are introduced by the specific dispersion properties of real glass. These are clearly visible in the lower left corner of the images.

In Figure 15a, a Petzval portrait lens design at aperture $f / 3.5$ is used. The design is well corrected for aberrations except for curvature of field and astigmatism. The resulting effects are shown for different image distances. Curvature of field is caused by the spherical surface of lenses. Thus, the real image surface is not planar but curved. Furthermore, due to the astigmatism, there is no image distance for which tangential and sagittal image features are in focus at the same time.

Fisheye Distortion In order to be able to build lenses with a field of view of 180 degrees, lens designers deliberately introduce distortions. Such designs are called Fisheye lenses and are amongst the most complex designs. Figure 16 shows two results, shot with different designs. Note the distortion of the window row near the ceiling, which is actually straight. This is impossible to achieve with a pinhole camera or thick lens model.

Depth of Field and Bokeh One obvious consequence of using a real lens design is a limited depth of field, especially for high speed lenses. Bokeh is the term for the visual appeal of the out-of-focus region. Here, the size and shape of the aperture is an important factor, as it influences the appearance of out-of-focus features. The effect is particularly noticeable in Figure 17 around the highlights in the back.

Lens Flares As already demonstrated in Figure 12a, our system renders lens flares due to interreflections. By saving the result of the forward light tracing pass, the lens flare can be examined separately from other effects. The example in Figure 12a clearly shows how a lens coating can suppress lens flare.
Diffraction Simulation Diffraction of incoming light at the aperture blades forms streaks perpendicular to the edges as can be seen in Figure 18. As noted above, diffraction effects can be simulated separately and simply added to the rest of the light transport. The resulting light streaks have a significant impact on the brightness perception of light sources.

Efficiency Discussion We benchmarked the lens stage of the rendering system and give values in 1.The eye path generation with a thin lens approximation took less than $2 \%$ for a regular scene. Because of the missing depth dimension of a thin lens and the possibility to sample the aperture area dicretly for path generation, there is no loss of rays during the passage. The Armadillo in our test setting has no environment geometry, which means most of the rays leaving the lens have no contribution, because they do not hit any surface at all. The thin lens computation time fraction increases by a factor of six, because no in-depth tree traversal and shading is done for most of the scene rays.

In a real lens scenario, these aspects are considerably different. The ray passage rate depends on all variables changing the optical path length and space of valid paths of the lens, among them focal length, sensor distance, glass properties, f-number and sensor dimensions. The numbers in the table are averaged values observed during our tests. The link between ray passage and f-number can be observed in all test cases. In combination with our simple pupil sampling method this lack can be completely eliminated. Additionally, the black pixels in the marginal region of a fish-eye shot can be identified, because their pupil has zero diameter. Of course, rendering time increases with higher ray passage, that is the main reason why the percentage of lens tracing time fraction decreases. In contrast to the global pupil sampling approach, the path computation fraction stays constant over all stop settings for one lens.

A fair comparison between thin lens and real lens is only meaningful for the pixel pupil sampling approach, where the ray passage rate gets optimized to a constant value within some limits. Two instances influence the passage rate in extreme conditions. A large distortion of the exit pupil makes the circle a rather bad approximation, which causes more wrong samples when using the pixel pupil, for example Rosier Gauss at $\mathrm{f} / 1$. This circumstance was already mentioned in 9 for fish-eye lenses was already explained. Second, as soon as the aperture is too narrow, the exit pupil image is no longer able to illuminate the whole sensor and produces pixels with zero diameter pupils, happening for Rosier Gauss at $\mathrm{f} / 8$. Another issue is the number of used samples for the pupil construction itself. The smaller the chosen aperture, the more samples are needed in theory to correctly determine the pixel pupil. Adaptive methods help to keep this problem small.

These numbers only consider pure image generation. Effects like diffraction or inner reflection cannot be combined 

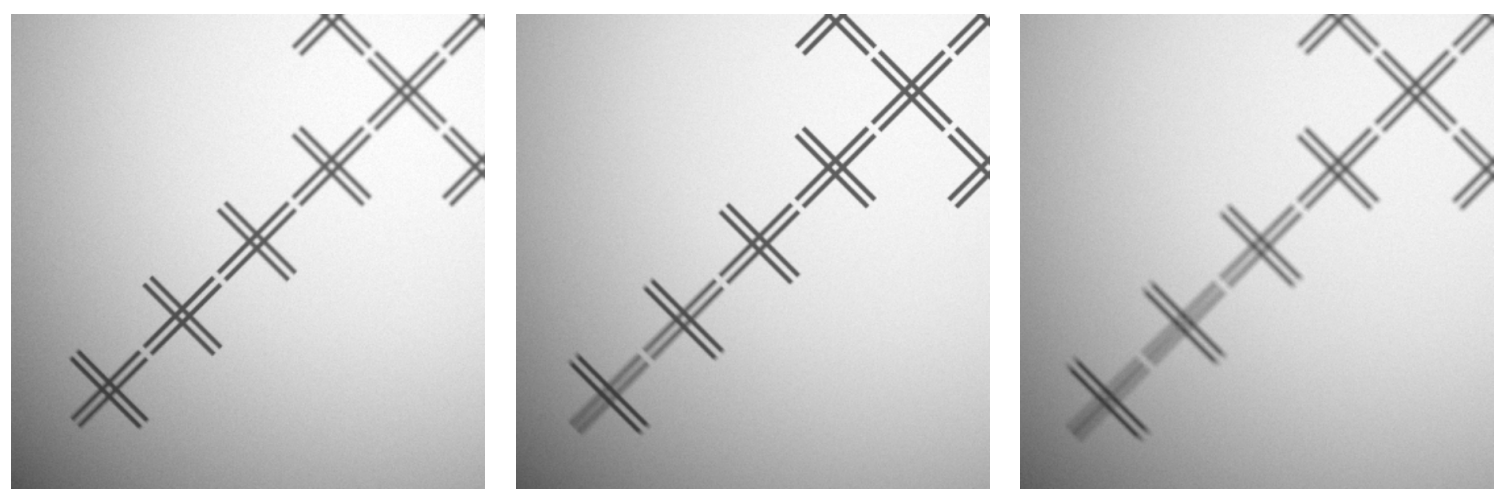

(a) In the rendering scenario observed for a Petzval lens at different image distances. Left: Visual evidence for field curvature. While the margins are sharp, the center is defocussed because the image plane is placed before the actual focal plane. Middle: Setting with the image plane moved towards the focal plane. The center gets focussed, but the margins lose sharpness. Right: This image made in the focal plane clearly reveals astigmatism. While the tangential structures in the marginal regions are rather focussed, the sagittal lines towards the center are blurred.
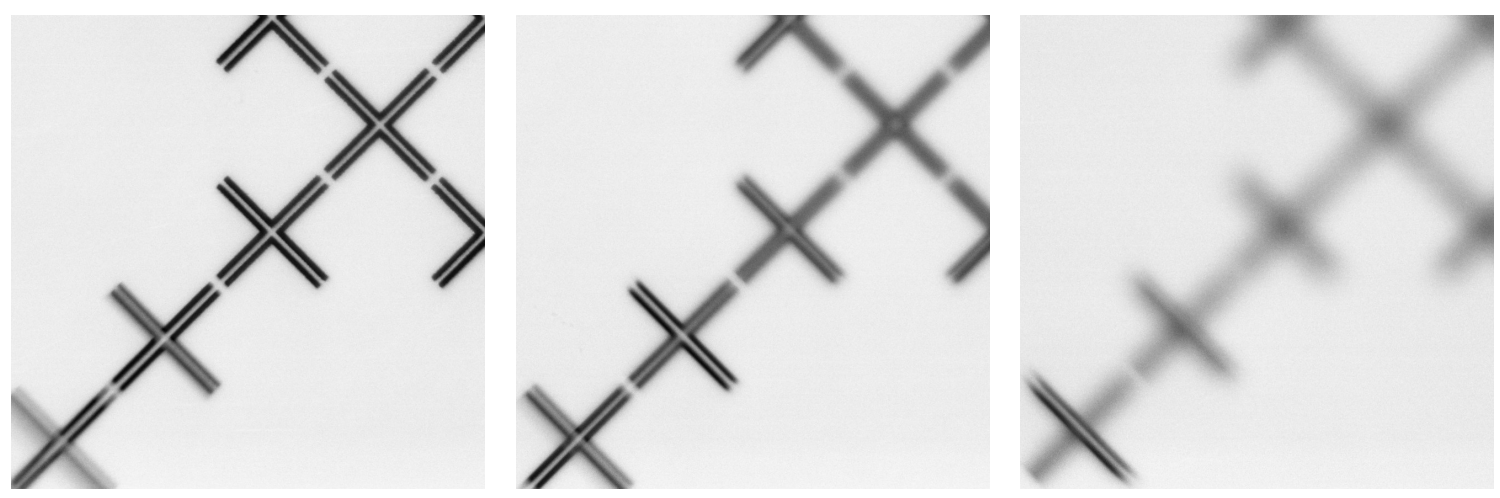

(b) Comparison photographs clearly showing the behaviour of astigmatism. Shot with a Zeiss Planar lens. By courtesy of Paul van Walree $[\mathrm{vW}]$.

Figure 15: Astigmatism and field curvature

with pixel pupil sampling, because the space of valid paths is much larger in these cases.

The lens simulation alone offers several opportunities to source the whole computation out to the GPU for example. The small number of analytic primitives makes an acceleration structure obsolete. Ray computation through the lens could massively benefit from parallel stream computation. This eliminates any CPU cycle costs for eye path generation from and to the sensor. The progressive rendering approach for including diffraction and flare effects only adds small extra rendering costs. The Armadillo example from the title page is given with absolute time numbers in 18 .

\section{Conclusion}

The correct optical simulation of all types of aberrations was introduced by the combination of spectral Monte Carlo light transport, real lens design data and the consideration of appropriate physical laws. These were additionally augmented by diffraction effects using the geometrical theory of diffraction.

The model could be generalised to other fields such as astronomy or microscopy and be used to simulate novel optical elements such as lenslet arrays, liquid lenses or phase plates. It is also possible to include polarization, interference and more general diffraction effects. As another prospect, simplified models for fast evaluation can be derived and verified against the reference output of our simulation.

\section{References}

[ALB] Alvaro Luna Bautista J. A.: http://www. 3drender.com/challenges - Lighting Challenge \#17 Natural History. [accessed 2009, October 14th].

[AM99] Aveneau L., MÉRiaux M.: Rendering polygonal scenes with diffraction account. In Proc. of the 16th Spring Conference on Computer Graphics (1999).

[CPC84] CoOK R. L., Porter T., CARPEnTER L.: Distributed ray tracing. Computer Graphics (Proc. SIGGRAPH '84) (1984), $137-145$. 

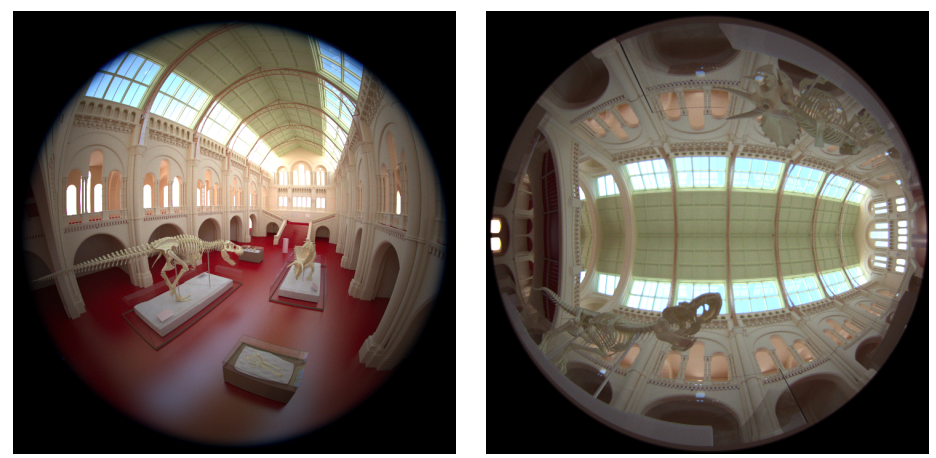

(a) Natural History scene [ALB], illuminated with a daylight environment. Rendered through a $14 \mathrm{~mm}$ Muller fisheye lens at $f / 5.6$ with $144^{\circ}$ field of view (left) and with a $12 \mathrm{~mm}$ Mitsuaki fisheye lens with $179^{\circ}$ (right).

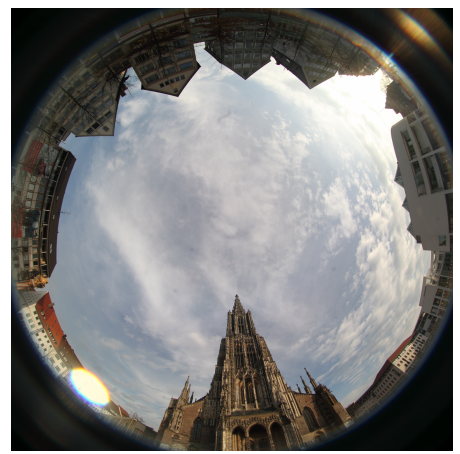

(b) Comparison panorama photograph of the Muensterplatz in Ulm.

Figure 16: Fisheye lenses produce characteristic panoramic images with $180^{\circ}$ field of view and above [Smi05].
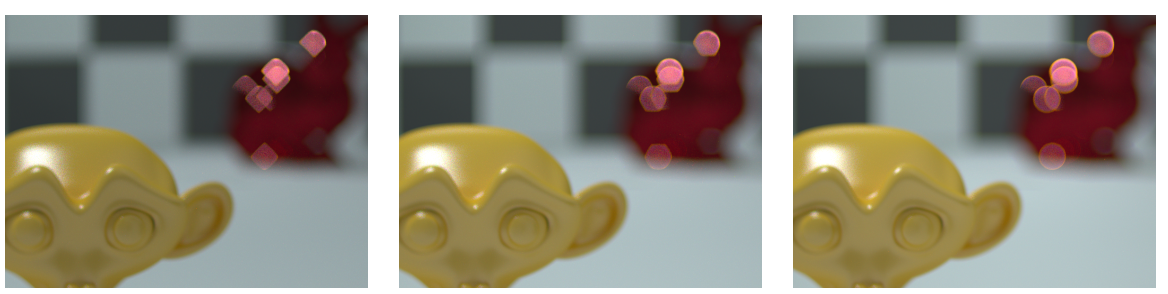

(a) These images were rendered through a Double Gauss lens by Rosier [Smi05] at speed $f / 1$. From top to bottom with 4,6 and 12 diaphragm blades. Besides becoming brighter by enlarging the aperture area the out-of-focus region of the image seems to become drawn much softer.

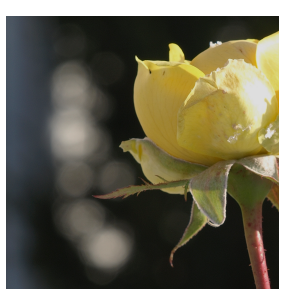

(b) The out of focus structures in a reference photograph taken with a 7-blade aperture.

Figure 17: Depth of field and influence of the aperture shape on out-focus-regions are clearly visible for high speed lenses.

[DCWP02] Devlin K., Chalmers A., Wilkie A., PurGATHOFER W.: Star: Tone reproduction and physically based spectral rendering. In State of the Art Reports, Eurographics 2002 (2002), pp. 101-123.

[Kaj86] KaJIYA J. T.: The rendering equation. Computer Graphics (Proc. SIGGRAPH '86) (1986).

[Kel62] Keller J. B.: Geometrical theory of diffraction. J. Opt. Soc. Am. 52, 2 (1962), 116-130.

[KMH95] Kolb C., Mitchell D., Hanrahan P.: A realistic camera model for computer graphics. Proc. of SIGGRAPH '95 (1995), 317-324.

[MK06] MichaEL KASS AARON LEFOHN J. O.: Interactive Depth of Field Using Simulated Diffusion on a GPU. Tech. rep., Pixar Animation Studios, 2006.

[MPM90] McNamara D. A., Pistorius C. W. I., MalHERBE J. A. G.: Introduction to the uniform Geometrical Theory of Diffraction. Artech House, 1990.

[PH04] PHARR M., HuMPhreys G.: Physically Based Rendering: From Theory to Implementation, 2nd ed. Morgan Kaufmann, 2004.

[PP06] Pedrotti F. L., Pedrotti L. S.: Introduction to Optics. Pearson Education (US), 2006.

[Ray02] RaY S. F.: Applied Photographic Optics, 3rd ed. Focal Press, 2002.

[Sch09] Schotт AG: Optical Glass - Data Sheets, 2009.

(c) 2010 The Author(s)

Journal compilation (C) 2010 The Eurographics Association and Blackwell Publishing Ltd.
[Shi00] ShIRley P.: Realistic Ray Tracing. AK Peters, Ltd., 2000.

[Smi05] Sмith W. J.: Modern Lens Design, 2nd ed. McGrawHill, 2005.

[Smi07] Sмiтh W. J.: Modern Optical Engineering, 4th ed. McGraw-Hill Professional, 2007.

[SSZG95] SPENCER G., Shirley P., Zimmerman K., GreENBERG D. P.: Physically-based glare effects for digital images. Computer Graphics 29, Annual Conference Series (1995), 325-334.

[Vea97] VEACH E.: Robust Monte Carlo Methods for Light Transport Simulation. PhD thesis, Stanford University, 1997.

[vS57] VON SEIDEL P. L.: Über die Theorie der Fehler, mit welchen die durch optische Instrumente gesehenen Bilder behaftet sind, und über die mathematischen Bedingungen ihrer Aufhebung. Abhandlungen der naturwissenschaftlichtechnischen Commission bei der Königlichen Bayerischen Akademie der Wissenschaften in München 1 (1857), 227-267.

[vW] VAN WALREE P.: http://www.toothwalker.org/ optics . htmI - Photographic Optics. [accessed 2009, October 14 th]. 
B. Steinert \& H. Dammertz \& J. Hanika \& H. P. A. Lensch / General Spectral Camera Lens Simulation

\begin{tabular}{|l|l||l|l|l|}
\hline \multirow{2}{*}{ Lens } & \multicolumn{1}{|l||}{ Setting } & Passage Rate & \multicolumn{2}{l|}{ Eye path generation time } \\
& & & MeshBox & Armadillo \\
\hline Thin lens & & $100 \%$ & $1.3 \%$ & $8.9 \%$ \\
\hline Tessar Brendel & f/2.8, GPS & $64.4 \%$ & $20.7 \%$ & $60.8 \%$ \\
4 elements in 3 groups & f/2.8, PPS & $87.6 \%$ & $17.8 \%$ & $55.7 \%$ \\
& f/8, GPS & $10.7 \%$ & $52.4 \%$ & $81.1 \%$ \\
& f/8, PPS & $85.7 \%$ & $17.7 \%$ & $55.7 \%$ \\
\hline Kimura wide-angle & f/2, GPS & $53.9 \%$ & $29.3 \%$ & $70.5 \%$ \\
7 elements in 6 groups & f/2, PPS & $81.8 \%$ & $26.4 \%$ & $67.0 \%$ \\
& f/5.6, GPS & $18.0 \%$ & $50.7 \%$ & $83.8 \%$ \\
& f/5.6, PPS & $84.9 \%$ & $26.9 \%$ & $66.8 \%$ \\
& f/16, GPS & $4.3 \%$ & $76.9 \%$ & $92.5 \%$ \\
& f/16, PPS & $86.1 \%$ & $27.0 \%$ & $67.2 \%$ \\
\hline Rosier Gauss & f/1, GPS & $40.7 \%$ & $34.9 \%$ & $76.3 \%$ \\
8 elements in 5 groups & f/1, PPS & $68.9 \%$ & $27.1 \%$ & $72.0 \%$ \\
& f/2.8, GPS & $6.1 \%$ & $73.4 \%$ & $91.5 \%$ \\
& f/2.8, PPS & $85.2 \%$ & $27.0 \%$ & $68.7 \%$ \\
& f/8, GPS & $0.9 \%$ & $93.0 \%$ & $96.1 \%$ \\
& f/8, PPS & $58.5 \%$ & $26.9 \%$ & $67.4 \%$ \\
\hline
\end{tabular}

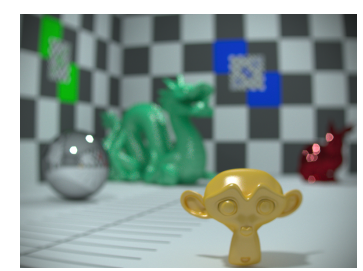

(a) MeshBox (approx 1.9M triangles) with Rosier Double Gauss lens.

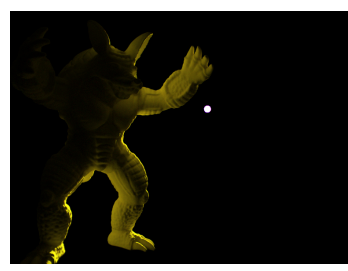

(b) Armadillo (approx. 350K triangles) in the dark with Kimura wide-angle lens.

Table 1: Presentation of rendering costs in relative numbers. GPS marks global pupil sampling settings, and PPS settings with our pixel pupil sampling approach. The values represent the part of rendering time needed for eye path generation per frame. Lens designs taken from [Smi05, Smi07]. We used approximately the same focal length of 55mm for all setups to have similar fields of view.

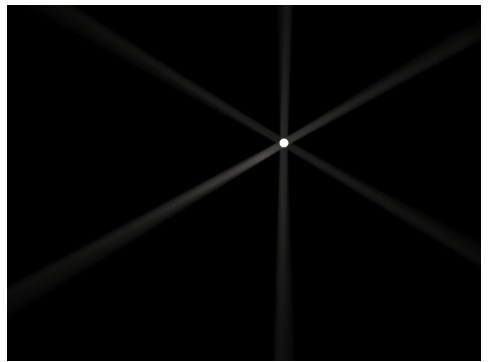

(a) Diffraction at the aperture simulated in a forward path tracing stage. Rendering took approximately 30 minutes.

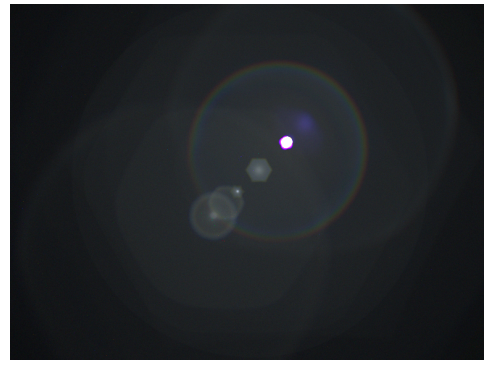

(b) The flare image with inner lens reflection consideration, slightly brightened. With forward path tracing, this result took 130 minutes to render.

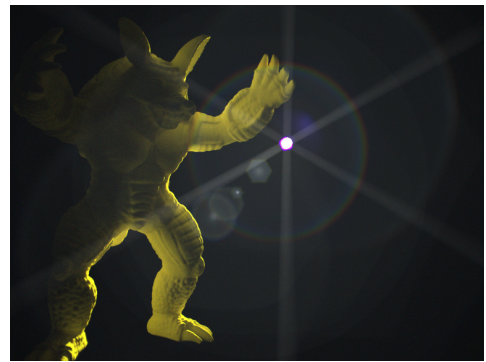

(c) Armadillo in the dark. Combined image by just summing up all previous images. Scene rendering without streaks or flares took 180 minutes.

Figure 18: An 800x600 sized example image stack of the proposed progressive approach with given absolute rendering times on the CPU. The multi-threaded Monte Carlo ray tracer works with a simple mono-ray SAH bounding volume hierarchy without any handmade optimizations. Direct light estimation was used for the backward as well as the forward path tracing stage. We used the GNU compiler collection $\mathrm{gcc}^{-4} .4$ and the machine was an 8-Core 2.5GHz Intel Xeon E5420. 